\title{
Principali test di stimolo e di inibizione per la patologia antero-ipofisaria: avvertenze per l'uso nella popolazione di età superiore ai 65 anni
}

\author{
Marina Caputo ${ }^{1,2} \cdot$ Chiara Mele $^{1} \cdot$ Alice Ferrero $^{1} \cdot$ Ilaria Leone $^{1} \cdot$ Paolo Marzullo $^{1} \cdot$ Flavia Prodam $^{1,2}$. \\ Gianluca Aimaretti ${ }^{1}$
}

Accettato: 14 maggio 2020 / Pubblicato online: 13 novembre 2020

(c) The Author(s) 2020

\begin{abstract}
Sommario La fisiopatologia dell'invecchiamento non è ancora stata del tutto chiarita sebbene numerosi filoni di ricerca siano attivi in questo ambito in considerazione dell'aumento della vita media della popolazione generale. Il sistema endocrino riveste un ruolo critico in questo contesto in quanto, durante l'invecchiamento, avvengono importanti modifiche nel pattern secretorio dell'asse ipotalamo-ipofisi e nella sua sensibilità ai meccanismi di feedback. Risulta pertanto evidente come, nell' ambito della quotidiana pratica clinica, sia necessaria un'interpretazione critica dei principali test endocrinologi per lo studio delle patologie ipofisarie nella popolazione over 65 , per la quale spesso non esistono cut-off età-specifici.
\end{abstract}

Parole chiave Aging · Test di stimolo - Test di inibizione . Asse corticotropo $\cdot$ Asse somatotropo $\cdot$ Invecchiamento e ormoni

\section{Introduzione}

La valutazione della funzione ipofisaria nel paziente anziano può essere complessa dal punto di vista diagnostico. Tuttavia, l'argomento è di grande attualità e rilevanza in consi-

Proposta da: Francesco Giorgino.

Materiale elettronico supplementare La versione elettronica di questo articolo (https://doi.org/10.1007/s40619-020-00794-0) contiene materiale supplementare, disponibile per gli utenti autorizzati.

$凶$ G. Aimaretti

gianluca.aimaretti@med.uniupo.it

1 Endocrinologia, Dipartimento di Medicina Traslazionale, Università degli Studi del Piemonte Orientale, Novara, Italia

2 Dipartimento di Scienze Mediche, Università degli Studi del Piemonte Orientale, Novara, Italia derazione dell'aumento della vita media nella popolazione generale e della maggiore incidenza di patologie, quali gli adenomi ipofisari, che comportano l'avvio del work-up diagnostico endocrinologico. Tale aumento di incidenza è dovuto al miglioramento e alla maggiore disponibilità di tecniche diagnostiche a elevata sensibilità, nonché al costante progredire delle conoscenze scientifiche in tale ambito.

Nello scenario attuale è necessario implementare la conoscenza della fisiologia e della fisiopatologia ipofisaria nell'invecchiamento (aging), con l'obiettivo di stabilire eventualmente specifici cut-off utili per la diagnostica in questi pazienti. Nella presente rassegna verranno descritti i meccanismi fisiopatologici da tenere in considerazione durante l'interpretazione dei test di stimolo o inibizione più frequentemente utilizzati nella normale pratica clinica [1].

\section{Aging e sistema endocrino}

L'invecchiamento è un fenomeno fisiologico caratterizzato da numerosi cambiamenti che avvengono in tutte le cellule e i tessuti, con conseguenze variabili e con caratteristiche specifiche per ciascun organo e apparato. Oltre ai meccanismi intrinseci che includono alterazioni cellulari legate allo stress ossidativo, alla metilazione e acetilazione del DNA, al cambiamento della lunghezza dei telomeri, così come al numero e alla funzione delle cellule staminali [2], sta emergendo il ruolo dello stile di vita e dei fattori ambientali. Il risultato finale dipende dalla somma e interazione delle alterazioni suddette e dalla capacità residua di riparazione delle cellule [3].

Per quanto riguarda le alterazioni del sistema endocrino, è necessario distinguere tra le modifiche ormonali connesse all'età (Fig. 1) e quelle conseguenti alle patologie ad alta prevalenza. Durante l'invecchiamento, avvengono modifi- 


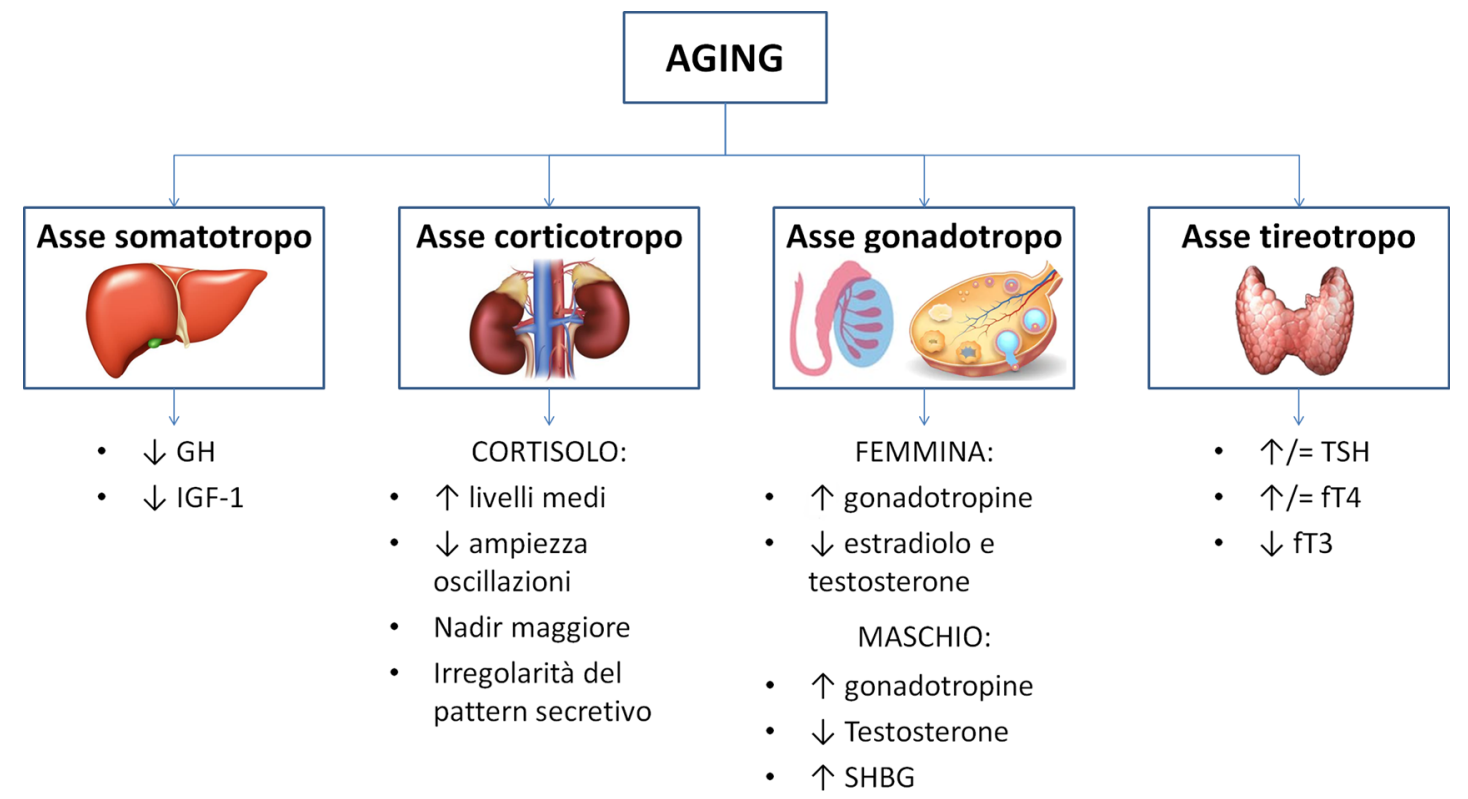

Fig. 1 Principali modifiche degli assi ormonali associate all'invecchiamento

che nel pattern secretorio degli assi ipotalamo-ipofisi e nella loro sensibilità ai meccanismi di feedback. L'invecchiamento si accompagna a profonde modificazioni dell'assetto neuroendocrino con una progressiva riduzione della sintesi e/o azione periferica di ormoni anabolici, come l'ormone della crescita $(\mathrm{GH})$ e gli ormoni gonadici, e l'aumento della sintesi di altri ormoni ad azione catabolica, come il cortisolo.

Nei paragrafi successivi verranno indagati gli aspetti fisiologici dell'invecchiamento relativi all'asse corticotropo e somatotropo, per poi focalizzarsi sui test di stimolo e di inibizione maggiormente utilizzati nella pratica clinica. In questa rassegna non verranno trattati l'asse tireotropo e gonadico in quanto, nel contesto delle alterazioni di tali assi nell'anziano, i test dinamici vengono scarsamente utilizzati.

\section{Asse corticotropo}

L'attività basale dell'asse ipotalamo-ipofisi-surrene (HPA) mostra un chiaro ritmo circadiano: il profilo delle 24 ore dei livelli periferici di ormone adrenocorticotropo (ACTH) e di cortisolo sono paralleli e sono caratterizzati da elevati livelli nel primo mattino (acrofase), un declino durante il giorno, con un prolungato periodo caratterizzato da bassi livelli (quiescenza) con un nadir intorno a mezzanotte e un rapido aumento successivo [1, 4].

In soggetti normali, i livelli medi di cortisolo nelle 24 ore si mostrano progressivamente crescenti dalla seconda all'ottava decade, con un' ampiezza minore delle oscillazioni. $\mathrm{Nel}$ soggetto anziano, il ritmo circadiano di ACTH e cortisolo è ridotto nell'ampiezza assoluta a causa dell'elevato nadir di cortisolo a fine giornata. Inoltre, il picco di cortisolo e il nadir sono più precoci al mattino (di circa 2 ore).
Studi nell'animale hanno dimostrato che tali modifiche riflettano l'attenuazione del feedback negativo, derivante dalla diminuita espressione dei recettori dei glucocorticoidi e dei mineralcorticoidi a livello dell'ippocampo e all'attivazione dei neuroni CRH e vasopressina (AVP) secernenti a livello ipotalamico [5].

Alla luce dei meccanismi descritti, non sorprende come l'iperattività dell'asse HPA nel soggetto anziano possa essere associata ad alcuni disturbi psichiatrici, tra cui la depressione, che risulta essere molto frequente nel paziente anziano. Cambiamenti neuroendocrini ben documentati nei soggetti depressi, includono un aumento dei livelli di cortisolo (in particolare al momento del cortisolo nadir) e aumento dei livelli di $\mathrm{ACTH}$, modifiche di per sé già tipiche dell'anziano [4].

I cambiamenti relativi all'invecchiamento dell'asse HPA sono più evidenti nelle donne rispetto agli uomini, e, come detto, nei pazienti con malattie neurologiche e psichiatriche. Diabete mellito, infiammazione cronica, ipertensione arteriosa, apnea ostruttiva del sonno e polimorfismi genetici possono inoltre aumentare il rilascio di ACTH e, conseguentemente, di cortisolo [5]. Se i cambiamenti nei modelli di secrezione di cortisolo siano dovuti all'invecchiamento di per sé o se invece riflettano altri effetti legati alla senescenza (quali la presenza di infiammazione di basso grado, l'alterazione delle caratteristiche del sonno o cambiamenti nello stato sociale o emotivo), rimane ancora da chiarire.

\section{Asse somatotropo}

L'attività dell'asse GH-Insulin-like growth factor (IGF-I) varia durante la vita ed è ridotta nell'invecchiamento. La secrezione spontanea del GH è particolarmente spiccata alla 
nascita, si riduce successivamente a valori costanti che rimarranno tali sino all'inizio della pubertà, periodo in cui si verifica un nuovo incremento dovuto all'aumentata ampiezza dei picchi secretori. Nell'adulto, il rilascio giornaliero di GH subisce un costante declino, che risulta più accentuato nell'uomo rispetto alla donna, probabilmente da correlare al ruolo degli ormoni sessuali e alle loro modifiche età-correlate [6]. Nell'età senile, infine, si assiste a un'ulteriore riduzione della secrezione circadiana di GH (circa il $14 \%$ per decade) con decremento della frequenza e dell'ampiezza dei picchi associato a una riduzione della permanenza ormonale in circolo [6].

È ormai riconosciuto come la ridotta secrezione di GH nell'invecchiamento rifletta principalmente variazioni nel controllo ipotalamico della secrezione somatotropica, ovvero l'aumento dell'attività somatostatinergica e la ridotta attività del GH Releasing Hormone (GHRH) [6, 7]. Inoltre, è stata osservata nell'anziano una riduzione delle proteine leganti il GH e, quindi, una diversa produzione o clearance del GH recettore [8]. Pertanto, le concentrazioni medie di GH nelle 24 ore, unitamente ai livelli di IGF-I e IGF Binding Protein (BP)-3, sono inferiori negli anziani rispetto a soggetti giovani [6].

Questa evidenza è ormai consolidata e ha portato oggi alla definizione di range di normalità dei livelli di IGF-I corretti per fascia di età, favorendo una migliore interpretazione dei disordini ormonali dell'asse GH-IGF-I in età geriatrica.

Un ruolo ulteriore nella ridotta attività dell'asse somatotropo potrebbe essere legato a variazioni dello stile di vita, compresi la riduzione dell'esercizio fisico e dell'alimentazione. È stato proposto il termine "somatopausa", che definisce in senso più ampio quell'insieme di alterazioni cliniche correlate all'invecchiamento, quali sarcopenia, perdita di massa ossea e incrementata adiposità viscerale con insulinoresistenza, che potrebbero riconoscere nella riduzione della secrezione di GH una possibile causa determinante.

\section{Test di stimolo}

La diagnosi dei deficit ipofisari nel paziente anziano si basa sulle stesse procedure diagnostiche utilizzate nel paziente adulto. Tuttavia, è necessario evidenziare l'assenza, per alcuni degli assi ipofisari, di parametri di riferimento normali età-correlati. In mancanza di tali informazioni, verranno fornite indicazioni di appropriatezza basate soprattutto sull'esperienza clinica e sulla conoscenza della fisiopatologia degli ormoni ipofisari.

\section{Deficit di ACTH}

L'insufficienza surrenalica secondaria è una patologia caratterizzata dalla mancata produzione di cortisolo da parte della corteccia surrenalica dovuta alla carenza ipofisaria di ACTH. Nonostante si presenti spesso con sintomi sfumati ad ogni età (affaticamento, scarso appetito, perdita di peso, nausea), la diagnosi tempestiva è di fondamentale importanza in quanto tale condizione può rapidamente portare al decesso del paziente per una crisi ipocorticosurrenalica. In età senile, in particolare, sintomi aspecifici quali astenia, dimagramento, nausea, vomito, ipotensione arteriosa e ipoglicemia possono porre problemi di diagnosi differenziale con altre patologie frequenti durante l'invecchiamento, in particolare le malattie neoplastiche, la depressione e la malnutrizione.

Posto il sospetto clinico di insufficienza surrenalica, sono necessari test biochimici per confermare la diagnosi. Il test diagnostico di primo livello consiste nel dosaggio del cortisolo sierico basale mattutino: livelli di cortisolo basale $<3 \mu \mathrm{g} / \mathrm{dl}$ sono indicativi di ipocortisolismo, mentre livelli $>15 \mu \mathrm{g} / \mathrm{dl}$ permettono di escluderlo. Nel caso vengano, invece, riscontrati valori di cortisolo compresi tra 3 e $15 \mu \mathrm{g} / \mathrm{dl}$, diviene necessario effettuare un test di stimolo [9].

Il test all'ipoglicemia insulinica (ITT) è considerato il gold-standard per la diagnosi perché caratterizzato da elevata sensibilità e specificità. Tuttavia, la necessità di raggiungere valori glicemici compatibili con una condizione di ipoglicemia sintomatica ( $<40-45 \mathrm{mg} / \mathrm{dL})$ ne sconsiglia l'utilizzo nell' anziano, per l'elevato rischio neurologico (convulsioni) e soprattutto cardiologico (aritmie e ischemia miocardica). Pertanto, il test con ACTH, proposto ormai in alternativa all'ITT, dovrebbe essere considerato la procedura d'elezione per la diagnosi di iposurrenalismo in età geriatrica [10]. Le ultime linee guida dell'Endocrine Society suggeriscono di effettuare il test con ACTH a basse dosi $(1 \mu \mathrm{g}) \mathrm{o}$ a dosi standard (250 $\mu \mathrm{g})$ (Tabella 1) [9]. Una recente metaanalisi su 1209 pazienti adulti, ha dimostrato un' accuratezza diagnostica simile per i due test, che risulta essere moderata a causa della bassa sensibilità [11]. Nella popolazione geriatrica, il test con ACTH a basse dosi dovrebbe essere considerato di elezione per la diagnosi di iposurrenalismo secondario in quanto è stato dimostrato che la somministrazione di $1 \mu \mathrm{g}$ di ACTH e.v. non solo è capace di indurre in individui sani un incremento dei livelli di cortisolo sovrapponibile a quello indotto a dosi standard, ma assicura anche maggiore specificità [10]. Non esistono, tuttavia, studi di accuratezza nel paziente anziano.

\section{Deficit di GH}

Il deficit di GH (GHD) nell'adulto si può sviluppare in seguito a diverse condizioni patologiche e può essere l'esito di due differenti situazioni cliniche: il deficit di secrezione insorto in età adulta o il deficit insorto in età pediatrica e confermato al re-testing effettuato in età di transizione. Clinicamente, gli adulti con GHD tendono ad avere segni e sintomi di malattia sfumati, quali un aumento relativo della massa 
grassa e una diminuzione relativa della massa muscolare e, in molti casi, una riduzione dell'energia con una riduzione della qualità della vita. Tali sintomi sono aspecifici e spesso presentano notevole sovrapposizione con caratteristiche tipiche dell'età senile. Nonostante questo, dal momento che la precisione dei test dinamici dipende fortemente dalla probabilità pre-test di malattia, il work-up per GHD, anche nel soggetto anziano, dovrebbe essere intrapreso solo in particolari classi di soggetti ad alto sospetto clinico: 2) soggetti che presentano segni e sintomi di malattie ipotalamo-ipofisarie; 2) soggetti che sono stati sottoposti a irradiazione cranica per neoplasia o a trattamento di altro tipo per neoplasia intracranica; 3) pazienti con GHD insorto in età infantile, da sottoporre a re-testing; e 4) coloro che presentano anamnesi di trauma cranio-encefalico o di emorragia subaracnoidea [12-14].

La diagnosi di GHD in età adulta e anziana si basa, pertanto, sulla dimostrazione di una ridotta risposta di GH ai test di stimolo in un appropriato contesto clinico, in quanto la misurazione dei livelli IGF-I, così come di IGFBP-3, non distingue soggetti normali da soggetti con GHD, soprattutto nella popolazione in eccesso ponderale e in quella anziana $[12,14]$.

Il GHD deve essere attentamente distinto dalla riduzione della secrezione di GH che accompagna il normale processo di invecchiamento e l'obesità e l'importanza di un'accurata diagnosi di GHD nell'anziano si basa sulle evidenze di evitare trattamenti inutili e/o complicati da un punto di vista pratico in pazienti già sottoposti a multiple terapie farmacologiche.

L'ITT rappresenta il gold standard per la diagnosi di GHD nell'adulto secondo le Linee Guida della Endocrine Society del 2011 [14]. Tuttavia, come discusso nel precedente capitolo, il suo utilizzo è sconsigliato nel paziente anziano per il rischio di gravi effetti avversi. È da considerare, inoltre, che i meccanismi che portano all' aumento del GH durante tale test risentono essenzialmente dell'aumento dell'attività endogena del GHRH con concomitante riduzione del rilascio ipotalamico di somatostatina e dell'aumento del rilascio di catecolamine per attivazione alfa-adrenergica. In virtù di questi meccanismi, la risposta del GH all'ITT risente delle variazioni dell' asse somatotropo legate all'età [15].

Un test alternativo, di ampio utilizzo clinico, è il test GH$\mathrm{RH}+$ Arginina che risulta ben tollerato e presenta buona sensibilità e specificità [14]. È stato dimostrato che il test GHRH + Arginina distingue pazienti adulti con GHD dai soggetti normali con una sensibilità pari all'ITT, tenendo conto, ovviamente, che per ciascun test siano considerati appropriati cut-off [16]. Un importante punto a favore del GHRH + Arginina come test di prima scelta è il suo profilo di grande sicurezza e la mancanza di controindicazioni di rilievo, eccetto l'insufficienza renale cronica. Infine è importante ricordare come per il test GHRH + Arginina esistano oggi cut-off Body Mass Index (BMI)-relati. È stato infatti dimostrato che la risposta del GH a questo stimolo è negativamente associata al BMI: i cut-off che si associano alla miglior sensibilità e specificità sono 11,5 $\mu \mathrm{g} / \mathrm{l}, 8,0 \mu \mathrm{g} / \mathrm{l} \mathrm{e}$ $4,2 \mu \mathrm{g} / \mathrm{l}$ nella popolazione normopeso, sovrappeso e obesa, rispettivamente [16]. Tuttavia, poiché i test combinati stimolano sia l'ipotalamo sia l'ipofisi, il GHD dovuto a malattia ipotalamica può rimanere misconosciuto. Questo è esemplificato da studi su pazienti trattati con irradiazione cranica, nei quali l'ITT mostra la più alta sensibilità e specificità nei primi 5 anni dal trattamento radioterapico; se il picco di GH dopo il test GHRH + Arginina è normale in questa categoria di pazienti, potrebbe essere utilizzato l'ITT $[12,14]$.

Complessivamente, il test GHRH + Arginina dovrebbe essere il test di scelta per la diagnosi di GHD nel paziente anziano in quanto, oltre a un ottimo profilo di sicurezza, non fornisce risposte di GH correlate all'età mostrando, anzi, una risposta di GH simile durante tutta la vita di un individuo; questo sembrerebbe riflettere il fatto che la massima capacità somatotropa è conservata nell'invecchiamento, mentre il declino del GH legato all'età rifletterebbe le variazioni centrali nel controllo neuroendocrino della funzione ipofisaria [15].

Un altro test è il test con glucagone (GST) dove il cutoff di $3 \mu \mathrm{g} / \mathrm{l}$ mostra la migliore combinazione di sensibilità e specificità [14]. Non esistono dati validati che la risposta del GH al glucagone sia influenzata da sesso ed età, mentre è noto come l'effetto secretorio del glucagone sia ridotto nell'obesità. È un test generalmente ben tollerato sebbene sia possibile l'insorgenza di vomito per effetto di un sovradosaggio o di infusione rapida. Questo test è controindicato nei soggetti malnutriti o con glicemia a digiuno $>180 \mathrm{mg} / \mathrm{dL}$ e nei soggetti anziani, in relazione al possibile rischio di ipoglicemia tardiva.

Un altro test proposto è il GHRH + GH-Releasing Peptide-6 (GHRP-6), GH secretagogo sintetico, che è in grado di stimolare la secrezione di GH a livello ipotalamico e ipofisario e agisce come antagonista funzionale della somatostatina [17]. La risposta è considerata normale per un picco di $\mathrm{GH} \geq 20,0 \mu \mathrm{g} / \mathrm{l}$, mentre un picco di $\mathrm{GH} \leq 10,0 \mu \mathrm{g} / \mathrm{l}$ suggerisce la presenza GHD. In pazienti ad alto rischio di ipopituitarismo, che presentino un picco di GH compreso tra 10 e $20 \mu \mathrm{g} / \mathrm{l}$, la diagnosi definitiva dovrebbe essere formulata considerando l'appropriato contesto clinico o eseguendo un secondo test di stimolo [17]. L'effetto del GH dopo stimolo con GHRH combinato con un GH secretagogo come GHRP6 o ghrelin è fortemente dipendente dall'età, mostrando una ridotta attività con l'invecchiamento. Ciò probabilmente riflette i cambiamenti legati all'età nella regolazione primaria della secrezione di GH, che coinvolgono la compromissione dell'attività colinergica e l'ipertono somatostatinergico. Pertanto, la risposta al test non sembra essere affidabile nei soggetti anziani. 
Tabella 1 Test di stimolo per sospetto ipopituitarismo relativo all'asse corticotropo e somatotropo nel paziente adulto e anziano [9]

\begin{tabular}{|c|c|c|c|}
\hline Tipologia test & Procedura & Interpretazione (risposta normale) & $\begin{array}{l}\text { Controindicazioni nel paziente } \\
\text { anziano }\end{array}$ \\
\hline ACTH test $250 \mu \mathrm{g}$ & $\begin{array}{l}\text { Somministrare ACTH 1-24 } \\
\text { (cosyntropin) } 250 \mu \text { e e.v. o i.m. } \\
\text { Prelievi per cortisolo ai tempi: } 0 \text { ', } \\
30^{\prime}, 60^{\prime}\end{array}$ & Cortisolo picco $>18-20 \mu \mathrm{g} / \mathrm{dL}$ & \\
\hline ACTH test $1 \mu \mathrm{g}$ & $\begin{array}{l}\text { Somministrare ACTH } 1-24 \\
\text { (cosyntropin) } 1 \mu \mathrm{g} \text { e.v. } \\
\text { Prelievi per cortisolo ai tempi: } 0{ }^{\prime}, 30\end{array}$ & Cortisolo picco $>18 \mu \mathrm{g} / \mathrm{dL}$ & \\
\hline $\begin{array}{l}\text { Test all'ipoglicemia } \\
\text { insulinica (ITT) }\end{array}$ & $\begin{array}{l}\text { Somministrare una dose di insulina } \\
\text { regolare in bolo e.v. pari a 0,05-0,15 } \\
\text { U/Kg. } \\
\text { Prelievi per GH e/o ACTH e/o } \\
\text { cortisolo: ai tempi -30', 0', 30', 60', } \\
\text { 120' }\end{array}$ & $\begin{array}{l}\text { Raggiungimento di adeguata } \\
\text { ipoglicemia (glicemia }<40 \mathrm{mg} / \mathrm{dL} \text { ): } \\
\text { - GH picco }>3-5 \mu \mathrm{g} / \mathrm{L} \\
\text { - cortisolo picco }>18-20 \mu \mathrm{g} / \mathrm{dL}\end{array}$ & $\begin{array}{l}\text { Cardiopatia ischemica, malattie } \\
\text { cerebrovascolari, epilessia } \\
\text { Test rischioso negli anziani }\end{array}$ \\
\hline GHRH+Arginina & $\begin{array}{l}\text { Infondere } 0,5 \mathrm{~g} / \mathrm{Kg} \text { di arginina (fino a } \\
\text { un max di } 30 \mathrm{~g} \text { ) in } 100 \mathrm{cc} \text { di } \\
\text { soluzione fisiologica } \\
\text { Dose di GHRH: } 1 \mu \mathrm{g} / \mathrm{Kg} \text { (max } \\
100 \mu \mathrm{g} \text { ) } \\
\text { Al tempo 0' iniettare GHRH e.v. in } \\
\text { bolo e iniziare l'infusione di } \\
\text { Arginina da completare in } 30 \mathrm{~min} \\
\text { Prelievi per GH: ai tempi 0', 30', 45', } \\
60 \text { ', 75', 90', 105', 120' }\end{array}$ & $\begin{array}{l}\text { - } \mathrm{GH} \text { picco }>4 \mu \mathrm{g} / \mathrm{L} \\
\text { Meglio utilizzare cut-off BMI relati: } \\
\text { - } \mathrm{BMI}<25 \mathrm{~kg} / \mathrm{mq} \text {, } \\
\text { GH picco }>11,5 \mu \mathrm{g} / \mathrm{L} \\
-25<\mathrm{BMI}<30 \mathrm{~kg} / \mathrm{mq} \text {, } \\
\text { GH picco }>8 \mu \mathrm{g} / \mathrm{L} \\
\text { - } \mathrm{BMI}>30 \mathrm{Kg} / \mathrm{mq} \\
\text { GH picco }>4,2 \mu \mathrm{g} / \mathrm{L}\end{array}$ & $\begin{array}{l}\text { Neoplasie attive, retinopatia } \\
\text { diabetica, diabete mellito tipo } 1 \mathrm{e} \\
2 \text { scarsamente controllato, IRC, } \\
\text { falsi negativi nei soggetti } \\
\text { sottoposti a irradiazione del SNC }\end{array}$ \\
\hline Glucagone & $\begin{array}{l}\text { Somministrare glucagone } 1 \mathrm{mg} \\
(1,5 \mathrm{mg} \text { se peso corporeo }>90 \mathrm{Kg}) \\
\text { i.m. } \\
\text { Prelievi per GH e glicemia: ai tempi } \\
0^{\prime}, 30^{\prime}, 60^{\prime}, 90^{\prime}, 120^{\prime}, 150^{\prime}, 180^{\prime}, \\
210^{\prime}, 240^{\prime}\end{array}$ & $\begin{array}{l}\mathrm{GH} \text { picco }>3 \mu \mathrm{g} / \mathrm{L} \\
\text { Esistono cut-off correlati al BMI } \\
\text { (l'obesità può attenuare la risposta del } \\
\text { GH allo stimolo) }\end{array}$ & $\begin{array}{l}\text { Malnutrizione, glicemia a } \\
\text { digiuno }>180 \mathrm{mg} / \mathrm{dL}\end{array}$ \\
\hline
\end{tabular}

Fra i GHRP, va anche menzionato un farmaco recentemente approvato dall'EMA, macimorelin, che lega il recettore GHS-R1a [18]. Macimorelin è avvantaggiato dalla somministrazione orale $(0,5 \mathrm{mg} / \mathrm{kg})$, minore durata del test (90 minuti) e numero di prelievi (al basale e dopo 45, 60 e 90 minuti). Mostra una accuratezza paragonabile al GHRH + Arginina e ITT. La soglia diagnostica di GH per macimorelin è pari a $2,8 \mu \mathrm{g} / \mathrm{L}$, con la possibilità di impiegare una soglia di $\mathrm{GH}$ pari a $5,1 \mu \mathrm{g} / \mathrm{L}$ se la probabilità di GHD è elevata [19]. Il picco di GH dopo macimorelin è inversamente proporzionale al BMI. Come per altri GHsecretagoghi, l'esperienza con macimorelin nell'anziano è limitata e va usata con cautela in pazienti con manifestazioni proaritmiche.

Riassumendo, il test GHRH + arginina con cut-off BMI specifici sembra risultare il più sicuro e affidabile per la diagnosi di GHD anche in fase senile [15]. Bisogna, però, considerare che molto spesso il soggetto anziano, a causa della perdita della massa magra, potrebbe presentare un BMI apparentemente normale, ma un eccesso di tessuto adiposo viscerale associato a insulino-resistenza. Si deve, infatti, ricordare come nell'anziano il BMI non sia un indicatore suggerito per la distribuzione della massa corporea e, in particolare, dell' eccesso ponderale. Obiettivo per ricerche future è pertanto quello di valutare se i cut-off del test arginina + GHRH correlati alla circonferenza vita [20], piuttosto che al BMI, possano essere più accurati nel contesto dell'aging. Inoltre, in considerazione della modulazione da parte degli steroidi sessuali nella secrezione del $\mathrm{GH}$, potrebbero essere considerati cut-off sesso-specifici, in particolare nella popolazione anziana.

I test utilizzati nella pratica clinica e consigliati dalle linee guida sono riassunti nella Tabella 1.

\section{Test di inibizione}

Come nel caso dei deficit ipofisari, anche nelle sindromi da ipersecrezione ormonale la diagnosi nel paziente anziano si basa sulle stesse procedure utilizzate nel paziente adulto. In questo contesto, mentre nel caso dell' asse somatotropo sono stati stabiliti cut-off età-correlati almeno per quanto riguarda le IGF-I, nel caso dell' asse corticotropo non sono stati ancora definiti chiari valori di riferimento per la diagnostica nei soggetti anziani. 
Tabella 2 Test di inibizione per sospetto ipercortisolismo [22] e test di inibizione per sospetta ipersecrezione di GH [25]

\begin{tabular}{|c|c|c|c|}
\hline Tipologia test & Procedura & $\begin{array}{l}\text { Interpretazione } \\
\text { (risposta normale) }\end{array}$ & Controindicazioni \\
\hline Test di Nugent & $\begin{array}{l}\text { Somministrare } 1 \mathrm{mg} \mathrm{di} \\
\text { Desametasone ( } 2 \text { compresse da } \\
0,5 \mathrm{mg} \text { ) alle ore } 23: 00 \text {; misurare } \mathrm{i} \\
\text { livelli di cortisolo alle ore } 08: 00 \text { del } \\
\text { mattino successivo }\end{array}$ & Cortisolo $<1,8 \mu \mathrm{g} / \mathrm{dL}$ & $\begin{array}{l}\text { Attenzione ai farmaci che modificano } \\
\text { il metabolismo del desametasone. } \\
\text { Attenzione ai farmaci che aumentano } \\
\text { la CBG }\end{array}$ \\
\hline Test di Liddle I & $\begin{array}{l}\text { Somministrare desametasone } 0,5 \mathrm{mg} \\
\text { ogni } 6 \text { ore per } 48 \text { ore iniziando alle } \\
\text { 06:00 del primo giorno; misurare i } \\
\text { livelli di cortisolo alle ore } 08: 00 \mathrm{del} \\
\text { terzo giorno }\end{array}$ & Cortisolo $<1,8 \mu \mathrm{g} / \mathrm{dL}$ & $\begin{array}{l}\text { Attenzione ai farmaci che modificano } \\
\text { il metabolismo del desametasone. } \\
\text { Attenzione ai farmaci che aumentano } \\
\text { la CBG }\end{array}$ \\
\hline $\begin{array}{l}\text { OGTT } 75 \mathrm{~g} \text { (test orale di } \\
\text { tolleranza al glucosio) } \\
\text { per GH }\end{array}$ & $\begin{array}{l}\text { Somministrare glucosio } 75 \mathrm{~g} \text { per os } \\
\text { (già pronto in soluzione) } \\
\text { Prelievi per GH ai tempi: 0', 30', 60', } \\
\text { 90', 120' }\end{array}$ & $\mathrm{GH}$ nadir $<1 \mu \mathrm{g} / \mathrm{L}$ & Diabete mellito \\
\hline
\end{tabular}

\section{Ipersecrezione di cortisolo}

La sindrome di Cushing (CS) è il quadro clinico che deriva dall'esposizione cronica a un eccesso di glucocorticoidi. Nell'eziopatogenesi si riconoscono cause esogene (iatrogene) e cause endogene. Le prime sono le più frequenti e sono riconducibili all'utilizzo prolungato di farmaci corticosteroidei. Le seconde sono determinate da un'ipersecrezione spontanea di cortisolo da parte della corteccia surrenalica o di ACTH da parte di lesioni ipofisarie e sono caratterizzate dalla perdita del meccanismo di feedback negativo e dalla scomparsa del ritmo circadiano del cortisolo [21].

Nel contesto di un ipercortisolismo cronico, gli effetti dei glucocorticoidi in origine fisiologici per il mantenimento di un' adeguata pressione di perfusione in caso di shock settico o l'iperglicemia durante il digiuno, diventano maladattativi provocando la comparsa delle manifestazioni cliniche che caratterizzano la sindrome metabolica, quali l'obesità centrale, l'ipertensione arteriosa, l'intolleranza glicidica e la dislipidemia. Al quadro metabolico si affiancano altre complicanze, tra cui diversi disturbi della sfera neurologica e cognitiva: sindrome ansioso-depressiva, psicosi, deficit cognitivi e della memoria nonché disordini del sonno, di comune riscontro in questi pazienti ancor di più se in età avanzata.

Nei soggetti anziani i sintomi da ipercortisolismo possono pertanto sovrapporsi a condizioni di per sé frequenti quali il diabete mellito, l'ipertensione arteriosa, la perdita di massa ossea, le alterazioni cognitive e disturbi del ritmo sonno-veglia. Pertanto, durante l'invecchiamento, è particolarmente difficile distinguere tali alterazioni come patologia-specifica o semplicemente legate all'età.

Il dato laboratoristico di ipercortisolismo va interpretato alla luce di un quadro clinico appropriato. Esclusa la forma più frequente di ipercortisolismo iatrogeno, il primo step diagnostico comprende i test di screening, tra cui il test di inibizione con desametasone (1 mg overnight o test di Nugent; $2 \mathrm{mg} /$ die per 2 giorni o test di Liddle I) (Tabella 2). Viene considerata diagnostica per ipercortisolismo la mancata soppressione della secrezione di cortisolo $(>1,8 \mu \mathrm{g} / \mathrm{dL})$. Tra gli svantaggi riscontrati per questo test bisogna considerare che alcuni farmaci (assunti con frequenza nella popolazione anziana) sono in grado di interferire con l'assorbimento o il metabolismo del desametasone, agendo come modulatori del CYP3A4 e che condizioni cliniche associate a un aumento dei livelli circolanti di transcortina (CBG) possono causare falsi positivi. Inoltre, la clearance del desametasone può essere influenzata da alterazioni della funzione renale o epatica (anche queste condizioni cliniche frequenti nel soggetto anziano) [22].

Relativamente alle insidie diagnostiche nella popolazione anziana, non sono stati riscontrati cambiamenti nei livelli di CBG durante l'invecchiamento in soggetti normali e, in uno studio longitudinale su soggetti sani anziani, modifiche nei livelli di cortisolo totale riflettevano le modifiche nei livelli di cortisolo libero [23]. In generale, i risultati dei test di soppressione dell'asse ipotalamo-ipofisi-surrene mostrano livelli medi di cortisolo maggiori nei pazienti anziani paragonati ai giovani, a prescindere dallo stato di salute. Infatti, è stato dimostrato come esista una correlazione significativa tra i livelli di cortisolo post-desametasone e l'età [23]; in realtà, non tutti i lavori hanno dimostrato tale associazione ma la differenza nei risultati sembra essere fortemente influenzata dall'età media della popolazione in studio. Tra le ipotesi proposte per spiegare la perdita del feedback nei soggetti anziani, alcuni autori hanno ipotizzato un possibile ruolo di una inadeguata concentrazione di desametasone, sebbene tale evidenza non sembri supportata da studi in vivo [24] e di una possibile alterazione della sensibilità del recettore dei glucocorticoidi a livello centrale. A livello ippocam- 
pale, in particolare, dove sono presenti numerosi recettori per i glucocorticoidi, è stato dimostrato come la resistenza al desametasone possa essere legata alla perdita cellulare età-correlata.

Nonostante tali evidenze, non sono stati validati cut-off età-correlati per i test di soppressione al desametasone. Pertanto, nell'interpretazione dei risultati, in particolare nella popolazione anziana, è necessario integrare il dato di laboratorio al dato clinico, tenendo in considerazione farmaci interferenti (fenobarbital, fenitoina, carbamazepina), malassorbimenti e patologie concomitanti (depressione).

\section{Ipersecrezione di GH}

La diagnosi di acromegalia si basa sulla misurazione dei livelli circolanti di IGF-I [25]. In soggetti normali, l'IGF-I diminuisce con l'età; per tale motivo, i livelli di IGF-I devono essere interpretati secondo cut-off età- e sesso-correlati.

Livelli elevati di GH random di per sé non permettono di fare diagnosi di acromegalia a causa della natura pulsatile della secrezione di tale ormone. Viene quindi raccomandata la valutazione della soppressione di GH dopo il test da carico orale di glucosio (OGTT $75 \mathrm{~g}$ ) come indagine laboratoristica complementare di conferma o nei casi dubbi. Tale test misura i livelli di GH ogni 30 minuti fino a 2 ore dopo la somministrazione orale di $75 \mathrm{~g}$ glucosio. In corso di iperglicemia durante OGTT, la mancata soppressione dei livelli di $\mathrm{GH}<1 \mu \mathrm{g} / \mathrm{L}$ permette di confermare la diagnosi [25]. Dopo l'introduzione di saggi più sensibili (ultrasensibili, chemiluminescenza) per il dosaggio di GH, è stata suggerita l'adozione di un cut-off inferiore per il nadir di GH, ovvero $0,4 \mu \mathrm{g} / \mathrm{L}$, ma tale limite non è ancora consigliato dalle linee guida a causa della variabilità delle metodiche di dosaggio [25].

Una risposta anomala di GH all'OGTT può essere associata ad età avanzata. Premesso che i livelli basali di GH sono inferiori nel soggetto anziano e che tale trend è mantenuto nel paziente acromegalico, è stato dimostrato che anche il GH nadir post-OGTT correla negativamente con l'età. In una coorte di pazienti naive con acromegalia (19-77 anni), livelli basali di GH e IGF-I e GH nadir dopo OGTT risultavano inferiori nei pazienti anziani (età $>60$ anni) [26]. Nel paziente anziano, tuttavia, l'OGTT a volte non può essere eseguito a causa della maggiore incidenza di diabete mellito e il profilo diurno di GH potrebbe costituire un test alternativo. Anche in questo caso, l'età svolge un ruolo importante; infatti, in una coorte di pazienti sottoposti a intervento chirurgico per acromegalia, la valutazione della remissione di malattia anche tramite il profilo di GH ha dimostrato cut-off differenti nei pazienti anziani se paragonati ai pazienti giovani o di mezza età $(1,4 \mu \mathrm{g} / \mathrm{L}$ vs $2,3 \mu \mathrm{g} / \mathrm{L})$ [27]. L'età, pertanto, risulta essere un fattore fondamentale da considerare nella diagnosi e nella valutazione dell'attività dell'acrome- galia. Come nel normale invecchiamento, questo fenomeno potrebbe essere correlato a una riduzione dei livelli ipotalamici di GHRH e ad alterazioni delle concentrazioni plasmatiche di steroidi. Inoltre, i pazienti più anziani solitamente presentano un peso corporeo più elevato o un eccesso di grasso viscerale rispetto a pazienti acromegalici più giovani, che potrebbero influenzare negativamente la risposta di $\mathrm{GH}$ all'OGTT [28].

\section{Conclusioni}

La gestione delle patologie dell'asse ipotalamo-ipofisario in età senile rappresenta ancora oggi una sfida dal punto di vista clinico-diagnostico. I sintomi che caratterizzano la ridotta o l'eccessiva secrezione di uno o più ormoni ipofisari sono aspecifici e non facilmente distinguibili dalle fisiologiche modificazioni dello stato di salute tipiche di questa popolazione. La diagnosi si basa sulle stesse procedure e sugli stessi cut-off utilizzati nell'adulto, anche se, per la maggior parte delle funzioni ipofisarie, dovrebbero essere stabiliti parametri di riferimento età-correlati. Ulteriori studi sono necessari per valutare i cut-off relativi a ciascun test diagnostico per poter migliorare la gestione e il trattamento delle patologie ipofisarie nella popolazione geriatrica.

Funding Note Open access funding provided by Università degli Studi del Piemonte Orientale Amedeo Avogrado within the CRUICARE Agreement.

Conflitto di interesse Gli autori Marina Caputo, Chiara Mele, Alice Ferrero, Ilaria Leone, Paolo Marzullo, Flavia Prodam e Gianluca Aimaretti dichiarano di non avere conflitti di interesse.

Consenso informato Lo studio presentato in questo articolo non ha richiesto sperimentazione umana.

Studi sugli animali Gli autori di questo articolo non hanno eseguito studi sugli animali.

Nota della casa editrice Springer Nature rimane neutrale in riguardo alle rivendicazioni giurisdizionali nelle mappe pubblicate e nelle affiliazioni istituzionali.

Open Access This article is licensed under a Creative Commons Attribution 4.0 International License, which permits use, sharing, adaptation, distribution and reproduction in any medium or format, as long as you give appropriate credit to the original author(s) and the source, provide a link to the Creative Commons licence, and indicate if changes were made. The images or other third party material in this article are included in the article's Creative Commons licence, unless indicated otherwise in a credit line to the material. If material is not included in the article's Creative Commons licence and your intended use is not permitted by statutory regulation or exceeds the permitted use, you will need to obtain permission directly from the copyright holder. To view a copy of this licence, visit http://creativecommons.org/licenses/by/4.0/. 


\section{Bibliografia}

1. Melmed S, Koenig R, Rosen C et al (2019) Williams textbook of endocrinology, 14th edn. Elsevier, Philadelphia

2. Bokov A, Chaudhuri A, Richardson A (2004) The role of oxidative damage and stress in aging. Mech Ageing Dev 125(10-11):811826

3. Conboy IM, Rando TA (2005) Aging, stem cells and tissue regeneration: lessons from muscle. Cell Cycle 4(3):407-410

4. Tsigos C, Chrousos GP (2002) Hypothalamic-pituitary-adrenal axis, neuroendocrine factors and stress. J Psychosom Res 53(4):865-871

5. Veldhuis JD (2013) Changes in pituitary function with ageing and implications for patient care. Nat Rev Endocrinol 9(4):205-215

6. Iranmanesh A, Lizzaralde G, Veldhuis JD (1991) Age and relative adiposity are specific negative determinants of the frequency and amplitude of GH secretory bursts and of the half-life of endogenous GH in healthy men. J Clin Endocrinol Metab 73:1081-1088

7. de Gennaro Colonna V, Cella SG, Locatelli V et al (1989) Neuroendocrine control of growth hormone secretion. Acta Paediatr Scand Suppl 349:87-100

8. Maheshwari H, Sharma L, Baumann G (1996) Decline of plasma growth hormone binding protein in old age. J Clin Endocrinol Metab 81(3):995-997

9. Fleseriu M, Hashim IA, Karavitaki N et al (2016) Hormonal replacement in hypopituitarism in adults: an Endocrine Society clinical practice guideline. J Clin Endocrinol Metab 2101(11):3888-3921

10. Gasperi M, Aversa A (2017) Manuale di Endocrinologia Geriatrica. SICS, Milano

11. Ospina NS, Al Nofal A, Bancos I et al (2016) ACTH stimulation tests for the diagnosis of adrenal insufficiency: systematic review and meta-analysis. J Clin Endocrinol Metab 101(2):427-434

12. Ho KK, 2007 GH Deficiency Consensus Workshop Participants (2007) Lawson Wilkins Society, European Society of Endocrinology, Japan Endocrine Society, and Endocrine Society of Australia. Eur J Endocrinol 157(6):695-700.

13. Cook DM, Yuen KC, Biller BM et al (2009) American Association of Clinical Endocrinologists. American Association of Clinical Endocrinologists medical guidelines for clinical practice for growth hormone use in growth hormone-deficient adults and transition patients - 2009 update. Endocr Pract 15(2):1-29

14. Molitch ME, Clemmons DR, Malozowski S et al (2011) Evaluation and treatment of adult growth hormone deficiency: an Endocrine Society clinical practice guideline. J Clin Endocrinol Metab 96(6):1587-1609
15. Giordano R, Aimaretti G, Lanfranco F et al (2005) Testing pituitary function in aging individuals. Endocrinol Metab Clin N Am 34(4):895-899

16. Prodam F, Pagano L, Corneli G et al (2008) Update on epidemiology, etiology, and diagnosis of adult growth hormone deficiency. J Endocrinol Invest 31(9:Suppl:6-11

17. Popovic V, Leal A, Micic D et al (2000) GH-releasing hormone and $\mathrm{GH}$-releasing peptide- 6 for diagnostic testing in GH-deficient adults. Lancet 356(9236):1137-1142

18. Piccoli F, Degen L, MacLean C et al (2007) Pharmacokinetics and pharmacodynamic effects of an oral ghrelin agonist in healthy subjects. J Clin Endocrinol Metab 92(5):1814-1820

19. Garcia JM, Biller BM, Korbonits M et al (2018) Macimorelin as a diagnostic test for adult growth hormone deficiency. J Clin Endocrinol Metab 103(8):3083-3093

20. Di Somma C, Ciresi A, Amato MC et al (2015) Alteration of the growth hormone axis, visceral fat dysfunction, and early cardiometabolic risk in adults: the role of the visceral adiposity index. Endocrine 49(2):492-502

21. Lahera Vargas M, da Costa CV (2009) Prevalence, etiology and clinical findings of Cushing's syndrome. Endocrinol Nutr 56(1):32-39

22. Nieman LK, Biller BM, Findling JW et al (2008) The diagnosis of Cushing's syndrome: an Endocrine Society clinical practice guideline. J Clin Endocrinol Metab 93(5):1526-1540

23. Lupien S, Lecours AR, Schwartz G et al (1996) Longitudinal study of basal cortisol levels in healthy elderly subjects: evidence for subgroups. Neurobiol Aging 17(1):95-105

24. O'Brien JT, Schweitzer I, Ames D et al (1994) Cortisol suppression by dexamethasone in the healthy elderly: effects of age, dexamethasone levels, and cognitive function. Biol Psychiatry 36(6):389-394

25. Katznelson L, Laws ER Jr Melmed S et al (2014) Acromegaly: an endocrine society clinical practice guideline. J Clin Endocrinol Metab 99(11):3933-3951

26. Colao A, Amato G, Pedroncelli AM et al (2002) Gender- and agerelated differences in the endocrine parameters of acromegaly. $\mathbf{J}$ Endocrinol Invest 25(6):532-538

27. Colao A, Pivonello R, Cavallo LM et al (2006) Age changes the diagnostic accuracy of mean profile and nadir growth hormone levels after oral glucose in postoperative patients with acromegaly. Clin Endocrinol 65(2):250-256

28. Ambrosio MR, Gagliardi I, Chiloiro S et al (2020) Acromegaly in the elderly patients. Endocrine 68(1):16-31 